\title{
Introduction to the special issue on history development of solar terrestrial sciences including auroral sub-storms
}

\author{
N. Balan ${ }^{*}$ (E) G. Parks ${ }^{2}$, L. Svalgaard ${ }^{3}$, Y. Kamide ${ }^{4}$ and T. Lui ${ }^{5}$
}

\begin{abstract}
Solar terrestrial (ST) sciences started centuries ago and branched into different disciplines. Starting with naked eye to highly sophisticated novel experimental techniques, observations have revealed the secrets of the Sun, heliosphere, magnetosphere, plasmasphere, and ionosphere-atmosphere components of the ST system. Theories and theoretical models have been developed for the different components independently and together. World-wide efforts under different umbrella are being persuaded to understand the challenges of the ST system. The onset problem and role of $\mathrm{O}^{+}$ions in sub-storm physics are two issues that are hotly debated. The onset problem is whether sub-storm is triggered by magnetic reconnection in the tail region at 15-20 Re or by a current disruption at $\sim 12$ Re. The issue on $\mathrm{O}^{+}$role is whether $\mathrm{O}^{+}$ions affect the dynamics of sub-storms under magnetic storm and non-storm conditions differently. This special issue of Geoscience Letters contains a collection of 15 papers on the history and development of solar terrestrial sciences including auroral sub-storms. Over half of the papers are based on the presentations in a session on the same topic organized at the AOGS (Asia Oceania geosciences Society) General Assembly held in Singapore during 02-07 August 2015. The rest of the papers from outside the assembly also falls within the theme of the special issue. The papers are organized in the order of history and development of ST coupling, sub-storms, and outer heliosphere.
\end{abstract}

\section{Solar terrestrial coupling}

Apart from solar electromagnetic radiations, the solar wind discovered in 1950s (Parker 1958) and coronal mass ejections (CMEs) discovered in the 1970s (e.g., Hansen et al. 1971; Tousey 1973) are the most important players in solar terrestrial relationship. CMEs are the root cause of severe weather in Earth's space environment, and are now counted among the major natural hazards. In a review article, Gopalswamy (2016) identifies a number of key developments that preceded the discovery of whitelight CMEs. CMEs can drive shocks that cause large solar energetic particle (SEP) events, and can impact Earth's magnetosphere producing extreme geomagnetic storms, all of which pose danger to humans and their technology in space and ground. Geomagnetic storms discovered in

\footnotetext{
*Correspondence: balannanan@gmail.com

1 INPE, São José dos Campos, SP CEP 12227-010, Brazil Full list of author information is available at the end of the article
}

the 1700s, solar flares discovered in 1859, SEP events discovered in 1942, and shocks discovered in 1962 are now found to be closely related to CMEs.

The transmission of solar wind energy to the Earth's space environment is the most important aspect of solar terrestrial coupling, the initial process of which is the magnetic reconnection between interplanetary magnetic field (IMF) and magnetopause magnetic field. Kikuchi and Hashimoto (2016) review the processes involved in the transfer of energy from the magnetosphere to the ionosphere. It involves an electric circuit starting from a dynamo in the magnetosphere to the equatorial ionosphere via polar ionosphere. They show that from high latitude ionosphere, the electric potential and currents are transmitted near-instantaneously to low-latitude ionosphere by TM0 (zero-order transverse electromagnetic) mode waves in the Earth-ionosphere waveguide.

In the history of geomagnetism, geoelectricity, and space science, the use of ground magnetic records has 
demonstrated to be a powerful tool for monitoring the levels of overall geomagnetic activity. Different indices such as Dst, Kp, and Ap were proposed to express different aspects of geomagnetic field variations. In early 1980s, several research groups in Japan, Russia, Europe, and the US developed the so-called magnetogram-inversion techniques. In a review article, Kamide and Balan (2016) demonstrate how important it was to make full use of ground magnetic data. By applying the inverse methods, it is now possible to map a number of electrodynamic parameters in the polar ionosphere on an instantaneous basis.

Using the continuous Dst data available since 1957, Balan et al. (2016) show that the mean value of Dst during the main phase, called mean Dst ${ }_{\mathrm{MP}}$, is a unique parameter that can indicate the severity of space weather, while the conventional parameter DstMin is insufficient. All five extreme storms having high mean Dst $_{\mathrm{MP}}(\leq-250 \mathrm{nT})$, which corresponds to high amount of energy input in the magnetosphere-ionosphere system in short duration, are found associated with severe space weather that caused all known electric power outages and telegraph system failures. Lakhina and Tsurutani (2016) review the interplanetary and solar causes of the extreme storms in September 1859 and March 1989.

Fraser (2016) outlines the historical development of ST physics in Australia and its international connections over the years. ST research in Australia began in 1792 when Elisabeth Paul Edouard de Rossel measured the southern hemisphere geomagnetic field at Recherche Bay on the southern tip of Tasmania, proving the field magnitude and direction varied with latitude. From the early 20th century, Australian ST research concentrated on radio wave propagation and communication, which by the 1950s fed into the IGY (1958) in the areas of atmosphere and ionosphere physics, and geomagnetism, with some concentration on Antarctic research. The paper concludes with examples of specific research areas where Australia has excelled.

Liu et al. (2016) report the developments of ST sciences in Taiwan. The developments began with the operation of an ionosonde in 1952 and introduction of space physics courses in 1959. The activities speeded up since 1990s with the introduction of a VHF radar, ionospheric tomography network, HF Doppler sounder, digital ionosondes, GPS receiver network, and launching of a series of satellites such as ROCSAT-1 (renamed as FOROSAT-1) for ionospheric studies, FORMOSAT-2 for lightning-induced transient luminous events, and FORMOSAT-3 for vertical ionospheric electron density profiles on a globe scale. The paper also outlines the specific research areas where Taiwan has made important contributions.
A number of studies have been made recently to check if the ionosphere can be used as a precursor of earthquakes. Oyama et al. (2016) report the current status of the ionospheric precursor (IP) studies associated with large earthquakes (EQ). They also aim to prepare for a future EQ-dedicated satellite constellation, which is essential to demonstrate whether the ionosphere can be used for short-term EQ predictions. Abdu (2016) presents a review of the current understanding of the low-latitude ionospheric weather variations and electrodynamic processes. The dynamic state of the low-latitude ionosphere is largely controlled by the electric fields originating from dynamo actions by atmospheric waves propagating from below and solar wind-magnetosphere interaction from above. These electric fields cause structuring of the ionosphere in wide ranging spatial and temporal scales.

\section{Sub-storms}

Kazmer and Timár (2016) provide an English translation of the original Latin text describing the aurora borealis observed on September 10, 1580 by Marcello Squarcialupi, an Italian medical doctor working in the court of the Hungarian prince of Transylvania. His book, De coelo ardore, describes the aurora of September 1580 in great detail, providing exact data from his personal observations on the time, direction, shape, color, and variability. He invoked a rational explanation, bringing up only natural causes, and confronted these with the ruling Aristotelian view.

Recent emphasis on dipolarization fronts (DFs) has led to the impression that DFs play a significant role in bringing magnetic flux to the inner magnetosphere during sub-storms. Lui (2016a) investigates the amount of magnetic flux transport associated with DFs by examining the frozen-in field line condition (FIC) for previously reported $18 \mathrm{DF}$ events. The accumulated magnetic flux transport is only $\sim 0.1-2.2 \%$ of what is needed to account for magnetic flux increase in the near-Earth dipolarization during sub-storms, which casts doubt on the idea that DFs play a significant role in sub-storm dipolarization. Lui (2016b) examines the similarities and differences between the concepts of current disruption (CD) and magnetic reconnection (MR) involved in the energy transfer. From the similarities and differences, Lui concludes that $\mathrm{CD}$ can be viewed as a form of generalized MR (GMR) in which the requirement for a specific magnetic field geometry and its constraint on the plasma flow pattern are removed. Therefore, the $\mathrm{CD}$ concept has a broader scope in applications than MR concept alone.

McPherron (2016) describes the relation of the magnetospheric sub-storm (MS) to the sub-storm current wedge ( $\mathrm{SCW})$. MS is an organized sequence of events 
seen in the aurora near midnight. The most dramatic feature of MS is the sudden brightening and poleward expansion of the aurora tied to the dynamics in the geomagnetic tail. Intimately associated with this expansion is a westward electrical current flowing across the bulge of expanding aurora. This current is fed by a downward field-aligned current (FAC) at its eastern edge and an upward current at its western edge. This current system is called the SCW, which forms within a minute of auroral expansion. McPherron illustrates the involved scenario with a complex sub-storm and discusses some of the associated problems that are still not understood.

Nose (2016) studies the long-term variations in the plasma sheet ion composition and sub-storm occurrence over 23 years using the energetic ion flux $(9.4-212 \mathrm{keV} / \mathrm{e})$ data measured using Geotail satellite and sub-storm occurrences evaluated from the number of Pi2 pulsations detected at the Kakioka observatory. The results show that the plasma ion mass $(\mathrm{M})$ is $\sim 1.1 \mathrm{amu}$ during solar minimum and increases to 1.5-2.7 amu during solar maximum. The study using the unprecedentedly long dataset covering $\sim 23$ years provides additional observational evidence that heavy ions work to prevent the occurrence of sub-storms.

\section{Outer heliosphere}

The space around the Sun up to where its influence extends is the heliosphere. The Voyager Interstellar Mission (VIM) has recently crossed the outer boundary of the heliosphere or heliospheric termination shock (HTS) and entered the interstellar medium. Zank (2016) reports the observations of plasma and turbulence in the supersonic and subsonic solar wind in HTS and beyond made by VIM. These observations reveal that the plasma in the outer heliosphere and very local interstellar medium (VLISM) is composed of distinct thermal proton and electron and super-thermal pickup ions. Estimates of the collision frequencies show that the multi-component plasma is not collisionally equilibrated in either the outer heliosphere or VLISM.

\section{Authors' contributions}

All authors are the editors of this Special Issue of Geoscience Letters. They all contributed to this article which is an introduction to the Special Issue. All authors read and approved the final manuscript.

\section{Author details}

1 INPE, São José dos Campos, SP CEP 12227-010, Brazil. ${ }^{2}$ SSL, Berkeley, USA. ${ }^{3}$ HEPL, Stanford University, Stanford, CA, USA. ${ }^{4}$ Nagoya University, Nagoya 464-8601, Japan. ${ }^{5}$ JHU/APL, Laurel, MD 20723-6099, USA.

\section{Acknowledgements}

We thank all reviewers for the timely review of all papers in the Special Issue. We also thank AOGS for supporting the publication cost of the papers.

\section{Competing interests}

The authors declare that they have no competing interests.

Received: 16 April 2016 Accepted: 22 April 2016

Published online: 16 May 2016

\section{References}

Abdu MA (2016) Electrodynamics of ionospheric weather over low latitudes. GeoSci Lett. doi:10.1186/s40562-016-0043-6

Balan N, Batista IS, Tulasi Ram S, Rajesh PK (2016) A new parameter of geomagnetic storms for the severity of space weather. GeoSci Lett. doi:10.1186/ s40562-016-0036-5

Fraser B (2016) A brief history of solar-terrestrial physics in Australia. GeoSci Lett (in press)

Gopalswamy N (2016) History and development of coronal mass ejections as a key player in solar terrestrial relationship. GeoSci Lett. doi:10.1186/ s40562-016-0039-2

Hansen RT, Garcia CJ, Grognard RJM, Sheridan KV (1971) A coronal disturbance observed simultaneously with a white-light corona-meter and the $80 \mathrm{MHz}$ Culgoora radioheliograph. Proc Astron Soc Aust 2:57

Kamide Y, Balan N (2016) The importance of ground magnetic data in specifying the state of magnetosphere-ionosphere coupling: a personal view. GeoSci Lett. doi:10.1186/s40562-016-0042-7

Kazmer M, Timár G (2016) The first scientific description of aurora borealis - the 10 September 1580 event in Transylvania, recorded by Marcello Squarcialupi. GeoSci Lett (in press)

Kikuchi T, Hashimoto KK (2016) Transmission of the electric fields to the low latitude ionosphere in the magnetosphere-ionosphere current circuit. GeoSci Lett. doi:10.1186/s40562-016-0035-6

Lakhina S, Tsurutani BT (2016) Geomagnetic storms: Historical perspective to modern view. GeoSci Lett. doi:10.1186/s40562-016-0037-4

Liu J-Y, Chang LC-W, Chao C-K, Chu Y-H, Hau L-N, Huang C-M, Kuo C-L, Lee L-C, Lin C-H, Shue J-H, Su C-L, Tsai L-C, Lin C-H, Hsu R-R, Su H-T (2016) The fast development of solar terrestrial sciences in Taiwan. GeoSci Lett (in press)

Lui T (2016a) Dipolarization fronts and magnetic flux transport. GeoSci Lett. doi:10.1186/s40562-015-0032-1

Lui T (2016b) Comparison of current disruption and magnetic reconnection. GeoSci Lett. doi:10.1186/s40562-015-0031-2

McPherron RL (2016) Relation of the auroral substorm to the substorm current wedge. GeoSci Lett. doi:10.1186/s40562-016-0044-5

Nose M (2016) Long-term variations in the plasma sheet ion composition and substorm occurrence over 23 years. GeoSci Lett. doi:10.1186/ s40562-015-0033-0

Oyama K-I, Devi M, Ryu K, Chen C-H, Liu J-Y, Liu H, Bankov L, Kodama T (2016) Modifications of the ionosphere prior to large earthquakes-report from the lonosphere Precursor Study Group. GeoSci Lett. doi:10.1186/ s40562-016-0038-3

Parker EN (1958) Dynamics of the interplanetary gas and magnetic fields. ApJ 128:664

Tousey R (1973) The Solar Corona. In: Rycroft MJ, Runcorn SK (eds) Space research XIII. AkademieVerlag, Berlin, p 713

Zank GP (2016) Plasma physics of the outer heliosphere and very local interstellar medium. GeoSci Lett (in press) 